\title{
Tratamiento del tinnitus en hipoacusias neurosensoriales de curvas descendentes por modulación de los neurotransmisores con el uso de acamprosato: Experiencia clínica
}

\author{
Tinnitus treatment by neurotransmitter modulation in high frequency \\ sensorineural hearing loss using Acamprosate: A clinical experience
}

\author{
Alejandro Peña Martínez¹.
}

\begin{abstract}
RESUMEN
Introducción: $\theta$ tinnitus neurosensorial está correlacionado con numerosas patologías del oído interno y de su vía central y que pueden originarse en cualquier nivel de ésta; sin embargo todas estas noxas pueden manifestarse igual en corteza auditiva, como una sensación auditiva sin mediar un estímulo acústico externo, de tal manera que este mensajero común deben ser los neurotransmisores. Se han descrito dos en la vía auditiva aferente: el glutamato que es excitatorio y el GABA que es inhibitorio. Numerosos estudios revelan que el tinnitus neurosensorial se produciría por un desbalance de estos dos neurotransmisores con predominio excitatorio. $\theta$ acamprosato es un fármaco usado en el alcoholismo que actuaría modulando el equilibrio GABA-glutamato. Existe un sólo estudio publicado con el uso del acamprosato en el tinnitus con una mejoría o disminución de su intensidad en $80 \%$ o más de los casos.

Objetivos: Conocer la real utilidad del fármaco pues en este estudio, arriba mencionado, la evaluación fue sólo subjetiva y sin seguimiento.

Material y Método: Fueron estudiados 20 pacientes tratados por un mes efectuándose tinnitumetría, evaluación psicoemocional (THI) y seguimiento.

Resultados: De los 20 pacientes, en dos desapareció el tinnitus, en seis bajaron los niveles de la tinnitumetría en 5 dB o más con mejoría del $7 H$ en $50 \%$ o más y en doce pacientes la mejoría fue menor o ninguna.

Conclusiones: Sería posible modular los neurotransmisores de la vía auditiva aferente con éxito, en aquellos pacientes con tinnitus severo. Es un estudio original con un respaldo fisiopatológico que abre nuevas perspectivas terapéuticas,

Palabras clave: Tratamiento del tinnitus, Acamprosato, neurotransmisores, glutamato, GABA
\end{abstract}

${ }^{1}$ Hospital Regional de Talca 


\section{ABSTRACT}

Background: Sensorineural tinnitus is correlated to several inner ear and auditory pathway disorders. However, noxas originating from different auditory pathway levels may be similarly manifested in the auditory cortex. A likely candidate for these shared messengers are neurotransmitters. Two neurotransmitters have been described in the afferent auditory pathways: glutamate, which is excitatory, and GABA, which is inhibitory. Different studies have shown that sensorineural tinnitus is caused by an imbalance of these two neurotransmitters with an excitatory predominance. Acamprosate, a drug used in the treatment of alcoholism, might act modulating the balance glutamate-GABA It could have a similar effect in the auditive afferent pathway. Only one research study on the use of acamprosate for tinnitus treatment has been published, which a result of over $80 \%$ of either relief or disappearance of tinnitus.

Aim: To investigate the actual usefulness of Acamprosate in tinnitus treatments, since in the previously mentioned study the results were subjectively measured, with no follow up.

Methods: Twenty patients treated with Acamprosate during a period of one month were studied; tinnitus psychoacoustic and psychoemotional assessment (THI) were carried out.

Results: Out of twenty patients, in two tinnitus disappeared, in six, it was reduced in 5 or more $\mathrm{dB}$ and in these same patients $7 \mathrm{H}$ improved in $50 \%$ or more. In 12 patients there was a very slight improvement or no changes at all.

Conclusions: It would be possible to modulate successfully the neurotransmitters of the afferent auditory pathway in some patients suffering from severe tinnitus using Acamprosate. This is an original study with physiopathological bases, which offers new therapeutic perspectives.

Key words: Tinnitus treatment, acamprosate, neurotransmitters, glutamate, GABA.

\section{INTRODUCCIÓN}

日 tinnitus sensorioneural o acúfenos es un síntomaque está correlacionado con múltiples enfermedades del órgano de Corti y de la vía auditiva; estas diferentes patologías se pueden localizar en cualquier nivel de esta vía, desde el oído interno, hasta la corteza cerebral auditiva; sin embargo, todas ellas pueden manifestarse igual, como una sensación auditiva sin mediar un estímulo acústico externo, que es lo que define el tinnitus sensorioneural o subjetivo.

Estos hechos llevan a concluir, que todas estas posibles causas deben tener un mismo camino de expresión, que no puede ser otro que los neurotransmisores.

A respecto, se han descubierto sólo dos de ellos para la vía auditiva aferente, tanto a nivel periférico como central, el glutamato y el ácido gamma amino-butírico o GABA ; en cambio en la vía auditiva eferente se han pesquisado algunos otros además del glutamato y el GABA: la dopamina y la acetilcolina ${ }^{1}$.

日 glutamato es el neurotransmisor más abundante en el SNC, y tiene un efecto excitatorio, el GABA en cambio tiene el efecto contrario, constituyéndose en el principal neurotransmisor inhibitorio tanto a nivel central como periférico; curiosamente, este último, se sintetiza a partir del glutamato, a través de una enżma, la glutamatodescarboxilasa ${ }^{2-4}$.

Al respecto, la intensidad del impulso eléctrico que recorre la neurona depende del potencial eléctrico a través de la membrana neuronal; dicho potencial viene determinado por la diferencia de concentraciones iónicas dentro y fuera de la neurona. Tales concentraciones son controladas por canales iónicos situados en la membrana y que regulan el flujo de iones al interior de la célula Varios son los iones implicados, si bien son los iones $\mathrm{C}^{-}$y $\mathrm{Ca}^{++}$los más importantes. Los recepto- 
res postsinápticos GABA activan los canales iónicos, permitiendo el paso de $\mathrm{C}^{-}$hacia el interior de la neurona, provocando una hiperpolarización y frenando la capacidad de enviar un estímulo eléctrico; en cambio la activación de los receptores postsinápticos ionotrópicos glutámicos, particularmente, los NMDA (N-metil-D-aspartato) permiten la entrada de iones $\mathrm{Ca}^{++}$a la célula, despolarizando la membrana y facilitando el envío de los impulsos eléctricos ${ }^{4,5}$.

Numerosos estudios actuales sobreel origen del tinnitus sensorioneural, orientan a pensar, que éste se produciría por una alteración de la modulación o desequilibrio del sistema glutamato-GABA, en que las diferentes patologías actuarían como verdaderas "espinas irritativas", provocando una alteración persistente central del sistema neurotransmisor a tra vés de la neuroplasticidad con liberación excesiva de glutamato y/o predominio excitatorio de éste ${ }^{6-9}$.

Estudios en animales han demostrado que en el envejecimiento y en el trauma acústico crónico, se produce un aumento de la actividad central del glutamato en la vía auditiva aferente, que se debe ría a una baja en las moléculas transportadoras de éste por los astrocitos de la glía 6 .

Aros estudios han revelado que cualquier le sión en la vía auditiva periférica, provoca un descenso de la actividad de la descarboxilasa del ácido glutámico en el colículo inferior, con la consiguiente disminución del GABA y predominio excitatorio, con el consecuente aumento de la actividad del colículo, lo cual sería percibido en la corteza auditiva como acúfenos ${ }^{7}$ y que ha llevado a estos investigadores a definir el tinnitus como "el dolor de la vía auditiva", alteración que ha sido objetivada en seres humanos a través de la RMf y el PET ${ }^{10-13}$.

Por otro lado, el exceso de glutamato provoca el fenómeno llamado excitotoxicidad que puede provocar un daño neuronal por excesiva acumulación de $\mathrm{Ca}^{++}$intracelular y eventual muerte de ellas. La excitotoxicidad produce una sobreactivación de los receptores postsinápticos ionotrópicos NMDA ( $\mathrm{N}$-metil-D aspartato), mecanismo que es el más aceptado por los investigadores, como causa del tinnitus sensorioneural y de varias patologías neurodegenerativas. Este mismo hecho ha sido corroborado por la observación de excesiva liberación de glutamato desde las células ciliadas internas del órgano de Corti a consecuencia de trauma acústico o isquemia aguda ${ }^{14}$.

Así, de esta manera, si pudiésemos actuar farmacológicamente, modulando en forma selectiva, particularmente a nivel central, este sistema permanentemente alterado de la vía auditiva aferente, podríamos suprimir o disminuir el tinnitus, lo cual es un enfoque nuevo y original sobre el problema, pues la mayor parte de las terapias se han encaminado a tratar de suprimir la posible causa, y no el síntoma.

Existen numerosos fármacos que actúan sobre los neurotransmisores sea estimulándolos o inhibiéndolos, particularmente los neurolépticos, pero sólo muy pocos que pudiesen actuar más específicamente sobre el sistema GABA-glutamato alterado de la vía auditiva aferente, tanto a nivel central como periférico y que no tuviesen mayores efectos colaterales indeseables.

Al respecto, dos fármacos han concitado el interés de los investigadores el presente año, uno es la Gabapentina, compuesto GABA-mimético en que una reciente publicación, ha mostrado alentadores resultados en la mejoría del acúfenos al usar altas dosis, particularmente en el tinnitus secundario a trauma acústico crónico ${ }^{15}$. 日 segundo fármaco, y que es el motivo del presente estudio, es el acamprosato.

Este fármaco sintetizado hace ya algunos años, se ha usado para el tratamiento del alcoholismo, bajo la hipótesis que esta adicción se produciría por una desbalance central del sistema GABAglutamato y en el cual el acamprosato modularía la acción del glutamato, inhibiendo su captación por los receptores postsinápticos NMDA, en el sitio cerebral responsable de la adicción. Igual acción tendría sobre el sistema auditivo aferente tanto a nivel central como periférico ${ }^{16,17}$.

Existe actualmente sólo un trabajo, recientemente publicado en la literatura mundial, del efecto de este fármaco sobre el acúfenos. Se trata de un estudio de doble ciego de autores brasileños que reportan una mejoría o disminución del tinnitus en más del $80 \%$ de los pacientes tratados con acamprosato ${ }^{17}$. 
Sin embargo, la lectura crítica de este estudio, plantea varias dudas sobre estos resultados tan auspiciosos: primero, la evaluación del tinnitus fue sólo subjetiva, en la cual el propio paciente le ponía nota (del 1 al 10) al síntoma, por lo que no se puede eliminar totalmente el efecto placebo, aunque sea un estudio ciego; segundo, se efectuó en un grupo seleccionado y pequeño (25 pacientes) y tercero, el estudio no informó que pasó con los pacientes al suspender el tratamiento, pues debe recordarse que se está actuando sobre el síntoma y no sobre la causa, y bien podría pensarse que el tinnitus reaparecería al suspender la terapia.

\section{OBJETIVOS}

Así, el propósito del presente estudio fue tener una experiencia propia, y responder a algunas de estas interrogantes sobre la real utilidad del acamprosato en el tratamiento del acúfenos.

\section{MATERIAL Y MÉTODO}

Para ello, a todos los pacientes de esta experiencia clínica, se les efectuó tinnitumetría de tonos puros, además de una evaluación psicoemocional del tinnitus, según el test de incapacidad del acufenos de Newman y Jacobson conocido por las siglas THI (Tinnitus Handicap Inventory) en escala de 0 a $100^{18}$. A respecto, llamó la atención algo ya publicado por Fowler el año $1942^{19}$ en el sentido que la intensidad del tinnitus medido en $\mathrm{dB}$ era muy baja, respecto al síntoma que algunos pacientes describían como algo tan insoportable, lo cual planteala gran dificultad para su evaluación, resaltando la importancia de la evaluación psicoemocional del síntoma Todos los pacientes de este estudio, habían previamente recibido, y sin ningún resultado, tratamientos con flunarizina y nimodipino los menores de 65 años, y nimodipino y betahistina los mayores de 65 años, durante dos meses, con lo cual de alguna manera se atenuó o minimizó el eventual efecto placebo del acamprosato. La dosis usada fue de $333 \mathrm{mg}$ cuatro veces al día, y se les prescribió durante un mes.
Veinte pacientes pudieron completar el estudio, pues es un fármaco caro. Todos ellos tenían hipoacusia neurosensorial de curva descendente (4 pacientes hipoacusia neurosensorial leve, me nos de $45 \mathrm{db} ; 12$ pacientes moderada, menor de 60 db y 4 pacientes hipocusia severa mayor de $65 \mathrm{db}$ ). En $75 \%$ de los pacientes la hipoacusia era bilateral; en $30 \%$ de ellos el acúfenos era bilateral, acentuado particularmente en un oído. Las posibles etiologías de las hipoacusias fueron las siguientes: presbiacusia (4), hipercolesterolemia (3), trauma acústico crónico (4), patología laboral muy común en la séptima región por la gran cantidad de aserraderos, sordera súbita antigua (3), intoxicación por aminoglicósidos (2), y de posible causas múltiples metabólicas, pacientes diabéticos e hipertensos (4). La edad de los pacientes fue de 49 a 75 años con un promedio de 62 años.

A todos ellos se les efectuó audiometría tonal, tinnitumetría de tonos puros, LDL, y evaluación subjetiva del tinnitus, según protocolo, el día primero, el día 15 y el día 30. Todos los pacientes ingresados en el estudio, debían tener impedanciometría normal y un tinnitus continuo, persistente no fluctuante. La duración del acúfenos experimentado por los pacientes iba de 8 meses a 10 años.

\section{RESULTADOS}

\section{El día primero}

- En ninguno de ellos el tinnitus fue igual a las frecuencias del audiómetro sino parecido, agrupándose en 14 pacientes en que el acúfenos era parecido a las frecuencias altas (de 4000 a 8000 Hertz) y en 6 pacientes a las frecuencias medias (750 a 1500 Hertz).

- La intensidad promedio del tinnitus medido en el audiómetro fue de $12,5 \mathrm{~dB}$, y la escala psicoemocional promedio del acúfenos, según protocolo, fue de 60, llamando la atención que no había una exacta correlación entre ambas escalas. 


\section{Al día treinta}

1‥ En dos pacientes el tinnitus desapareció (10\%)

2‥ En seis pacientes (30\%) disminuyó significativamente, entendiéndose como mejoría, cuando la escala psicoemocional disminuía en más de $50 \%$ y la tinnitumetría disminuía $5 \mathrm{~dB}$ o más. No hubo una directa correlación de la mejoría entre las escalas subjetivas y las tinnitumetrías

3o. En 12 pacientes el acamprosato tuvo un efecto menor o ninguno sobre el acúfenos (menos del $50 \%$ en la escala psicoemocional y menos de 5 $\mathrm{db}$ en la tinnitumetría).

4․ Ningún paciente tuvo variación audiométrica ni aumento del tinnitus terminado el tratamiento.

50. Se ha hecho un seguimiento de 1 a 4 meses después de finalizada la terapia en los pacientes en los cuales desapareció o disminuyó el acúfenos y no se ha observado recaída.

60. Aquellos pacientes en que se obtuvieron los mejores resultados, fueron los que tenían el síntoma por menos tiempo (8 meses a dos años).

7․ La mejoría del tinnitus no tuvo relación con la intensidad de la pérdida auditiva experimentada por ellos, sino con su tiempo de evolución.

8․ - B acamprosato fue excelentemente bien tolerado, y ninguno sufrió los eventuales efectos colaterales de tipo digestivo descritos en la farmacología ${ }^{16}$.

9․ Los resultados positivos se observaban ya el día 15, con leve mayor mejoría al día 30.

\section{DISCUSIÓN}

Los resultados obtenidos en este estudio, con una mejoría o desaparición del síntoma de $40 \%$, fueron sólo la mitad de lo informado en la investigación brasileña ${ }^{17}$ aunque cabe señalar que ellos reportaron una mejoría, aunque leve, de $40 \%$ en el grupo con placebo. Esta diferencia puede deberse también a que ellos usaron, sólo una escala subjetiva (nota puesta por el propio paciente), lo que hace más difícil su correcta evaluación. Además el 50\% de los pacientes brasileños no habían recibido terapia previa, a diferencia de nuestro grupo, pues es sabido, que con los tratamientos convencionales con vasodilatadores se podría obtener hasta $30 \%$ de mejoría, según diferentes estudios ${ }^{20}$ y finalmente, en la experiencia brasileña se usó el acamprosato durante 3 meses, lo cual en nuestro medio fue muy difícil por el alto costo del medicamento y no contar con un apoyo externo. Aro hecho que llamó la atención en este estudio, descrito por varios autores, fue la discordancia entre la evaluación psicoacústica (tinnitumetría) y la psicoemocional (THI). La teoría neurofisiológica encuentra la respuesta a esta independencia de la sensación respecto a la intensidad del acúfenos; si bien el tinnitus tiene habitualmente su origen en la cóclea, su mantenimiento e incremento dependen de mecanismos centrales, particularmente, a nivel del colículo inferior, jugando además un rol muy importante en los factores psicoemocionales el sistema límbico, el sistema nervioso autónomo y el córtex prefrontal que involucran respuestas de alerta, y reacciones psicológicas de temor y ansiedad, y que explica la poca importancia de las características psicoacústicas del tinnitus respecto al componente psicoemocional ${ }^{21}$.

Observamos además en nuestros pacientes, algo también ya descrito en la literatura ${ }^{22}$ que consistió en que todos los pacientes eran muy precisos en sus respuestas referentes a la intensidad del tinnitus y no así respecto a la frecuencia de éste la cual les era muy difícil precisar.

\section{CONCLUSIONES}

1‥ La posibilidad de poder modular selectivamente la acción de los neurotransmisores en el tinnitus abre un nuevo camino en la terapia de este síntoma, y así parecen demostrarlo los resultados obtenidos, mostrando además perspectivas para futuros fármacos que pudiesen actuar más específicamente sobre el sistema GABA-glutamato alterado de la vía auditiva aferente.

$2^{\circ}$. Posiblemente el acamprosato a la luz de esta experiencia, no sea "el fármaco" para el tratamiento del tinnitus sensorioneural, pero podría 
quizás, tener su indicación, en aquellos pacientes en que han fracasado los medicamentos más usados del arsenal terapéutico habitual.

3o. No es posible saber a través de esta investigación, la dosis óptima de acamprosato y el tiempo que se requeriría para el control del tinnitus, así en el presente estudio se usó $75 \%$ de la dosis recomendada para el tratamiento del alcoholismo (los brasileños un 50\%). Tampoco sabemos el tiempo eficaz necesario para la corrección del síntoma, teniendo en consideración que el acúfenos crónico es una alteración demostradamente central por fenómenos de neuroplasticidad ${ }^{23,24}$ particularmente a nivel de colículo inferior ${ }^{25}$, y que pudiese tomar un largo plazo en modificarse; en nuestro estudio lo usamos durante un mes y los brasileños por tres meses ${ }^{17}$. Al respecto en la adicción alcohólica se recomienda un tratamiento de seis a doce meses.

4․ Falta aún mucho por conocer la neurofisiología y fisiopatología de los neurotransmisores, pues desconocemos actualmente, si el tinnitus se debería a una síntesis excesiva de glutamato, o a un defecto en su recaptación por los astrocitos, o a una disminución de la síntesis del GABA, pues cualquiera de estas alteraciones puede llevar a un desbalance excitatorio GABAglutamato del sistema auditivo aferente central y/o periférico. Además, también se conoce muy poco la acción que pudiese tener el sistema auditivo eferente sobre el tinnitus.

5‥ Así pues el tinnitus sensorioneural debe ser considerado como una alteración heterogénea, para la cual no hay aún una terapia universal, y continúa siendo un desafío para el médico.

6․ Fnalmente, el presente estudio, posiblemente tenga el interés de su originalidad, pues hay sólo una sola experiencia mundial publicada en la literatura, al respecto.

\section{BIBLIOGRAFÍA}

1. Oestreacher E, Wolfgang A, Feix D. Neurotransmision of the cochlear inner hair cell synapse-implications for inner ear therapy. $A d v$ Aorhynolaryngol 2002; 59: 131-9.

2. Gamma-Aminobutyric acid. En: http:// en.wikipedia.org/wiki/gamma-aminobutyric.

3. Gutamic acid. En: http:// en.wikipedia.org/wiki/ glutamate.

4. LASSE $K$, AME SCHOUSBOE, Helle W. The glutamate/GABA-glutamine cycle: Aspects of transport, neurotransmitter homeostasis and ammonia transfer. Jour Neurochemestry 2006; 98(3): 64-70.

5. Los neurotransmisores en general. En: http:// www.psicomag.com/neurobiological/LOS\%.

6. SANTARELI R. Mecanismi neurofisiologici alla base degli acufeni: effetti inaspettati della plasticità neuronale, En: Ouda D. Acufeni: Diagnosi e Terapia Piacenza. TorGraf ed, 2004; 11-26.

7. HerRálz C. Mecanismos fisiopatológicos en la génesis y cronificación del acufenos. Acta Otorrinolaringol Esp 2005; 56: 335-42.

8. BAUER CA. Mechanisms of tinnitus generation. Ourr Opin Aolaryngol Head Neck Sur 2004; 12(5): 413-7.

9. James H, Kyle D, Martin S. General Review of Tinnitus: Prevalence, Effects and Management. Jour Speech Lang Hearing Res 2005; 48: 120435.

10. EGGRMONT JJ. Tinnitus: neurobiological substrates. Drugs Discov Today 2005; 10 (19): 1283-90.

11. Kela A, SáncheZ T. Auditory deprivation, Inhibitory circuits and plasticity; Implications for the comprehension of Tinnitus and Hiperacusis. Arquivosdeorl 2005; 9(4): 341-50.

12. Moller AR. Similarities between severe tinnitus and chronic pain. J Am Acad Audiol 2000; 11(3): 115-24.

13. MeCH'R JR, SIGALOVSKY IS, GUINAN JJ, LEVINE RA. Lateralized Tinnitus Studied with Functional Magnetic Resonance Imaging: Abnormal inferior Colliculus Activation. J Neurophysiol 2000; 83: 1058-72.

14. Puo R, PU日 JL. Excitotoxicity, synaptic repair and functional recovery in the mammalian cochlea: a review of recent findings. Ann Acad Sci 1999; 884: 1249-54. 
15. Baugr C, Brozoski T. Effect of Gabapentin on the Sensation and Impact of Tinnitus. Laryngoscope 2006; 116(5): 675-681.

16. Sour F, RodríGuez O, Sär C. Acamprosato: Nuevo fármaco en el tratamiento de la dependencia alcohólica. Farm Hosp 1997; 21(1): 1-10.

17. AZEVEDO A, FGUeredo R. Tinnitus treatment with acamprosate: a double-blind study. $\mathrm{Br}$ Journal of Otorhynol 2005; 71: 618-23.

18. NeMman C, JacoBson G, SPITZR J. Development of the Tinnitus Handicap Inventory. Arch Atolaryngol Head Neck Sur 1996; 122(2): 143-50.

19. FomleR $\boxminus$. The illusion of loudness of tinnitus, its etiology and treatment. Laryngoscope 1942; 52: 275-85.
20. MocH P. Terapia dell'acufene e medicine delle evidenza En: Ouda D. Acufeni: Diagnosi e Terapia. Piacenza: TorGraf ed, 2004; 107-22.

21. HerRálz C. Acufenometría. En: http:/ www.acufenos.info.com/acufenometria.html.

22. VERNON J. Relief of tinnitus by masking treatment. En: English G Aolaryngology Philadelphia. Ed Lippincott Co 1988: chap 53(1): 1-21.

23. Molter AR. Neural plasticity in tinnitus. Prog Brain Res 2006; 157: 365-72.

24. MURRI A, ODA D. La neuroplasticità nel sistema uditivo. En: Ouda D. Acufeni: Diagnosi e Terapia. Piacenza. TorGraf ed, 2004; 27-40.

25. FAINGOLD C. Role of GABA abnormalities in the inferior colliculus. Pathophysiology-audiogenic seizures. Hear Res 2002; 168(1-2): 223-37. 